\title{
Comentarios a la ponencia "Carcinoma intra-ipitelial del cervix uterino"
}

\author{
Profesor Pedro Nel Cardona C., doctores Gustavo Isaz? M., Gon. \\ zalo Vásquez V., Fernando Cardona A.
}

De la Cátedra de Ginecología de la Universidad de Antioquia.

La Convención Nacional de Obstetricia y Ginecologia ha de. signado como comentaristas de la Ponencia "Carcinoma Intra. epitelial del cervix uterino" presentada por la Delegación de Bo. gotá (Instituto Nacional de Cancerología), a la Delegación de Medellín.

Nosotros gustosos haremos el comentario del excelente traba_ jo presentado por los doctores Guillermo López Escobar, Luis A. Urdaneta, Enrique Darnalt, Germán Jordán, Hernando Osorio, Hugo Quijano y Jaime Salazar.

Es nuestro espíritu al hacer el presente comentario, el poner de relieve, dándole así un mayor realce las magníficas partes de la ponencia "Carcinoma Intra_epitelial del cêrvix uterino", y a la vez, presentar algunos puntos de vista, referentes al carcino. ma intra_epitelial; con la sola intención de mostrar el mismo pro. blema desde un ángulo diferente, para asi poder tener una visión de mayor profundidad semejante a la que vemos en la pantalia euando una imagen ha sido captada por dos cámaras en diferen. te ángulo.

La ponencia como bien la acaban de escuchar, ha sido divi. dida en tres partes: la primera en donde se presenta un resumen del cuadro histológico del cervix normal y patológico, y la inves. tigación del glucógeno; la segunda en donde se presenta y discu. te el resumen de los carcinomas intra_epiteliales hallados, y la tercera en donde se hacen comentarios y se sacan conclusiones relacionadas con el diagnóstico y la conducta que ha de seguirse en el carcinoma intra_epitelial.

Comenzaremos comentando, como es lógico, la primera par. te, es decir, la referente al cuadro histológico normal y patológi. co y a la investigación del glucógeno.

Los ponentes nos definieron en forma clara y académica los epitelios normales que se encuentran en el cervix, lo mismo que los estados de metaplasia, leucoplasia, anaplasia regenerativa, hi. 
poplasia, y las modificaciones del epitelio cervical en el embara. zo; en lo referente a las hiperplasias vimos que la clasificación seguida por nuestros ilustres y gentiles colegas fue la de las es_ cuelas americanas y francesas; que nosotros consideramos co. rrecta, lógica y acertada; sin embargo, creemos que la escuela alemana encabezada por Hinselmann, en lo referente a la c'asifi. cación de los epitelios atípicos, merece un breve recordatorio en nuestro comentario, ya que ella nos presenta los mismos epitelios pero catalogados en distinta forma, quizá un poco más didáctica, que no vacilamos en exponer, pues creemos que con ello cumpli_ mos en parte con nuestros deseos al presentar estos tejidos atípi. cos-desde un ángulo diferente (Clasificación tomada de "Atlas de Colposcopia de Gustav Mestwerdt").

Clasificación del epitelio atípico con tendencia querantinizan. te del cervix de Hinselmann:

Epitelio atípico simple con tendincia queratinizante.

= En extensión de superficie:

- Rúbrica I. - Discontinuidad neta con e? ıpitelio normal. Estra. tificación análoga a la piel: células basales (en general una ca_ pa), estratum germinativo ( 2 o 3 capas), estratum espinoso (mu_ chas capas), estratum córneo (o bien queratinisación verdadera o bien paraqueratosis) .

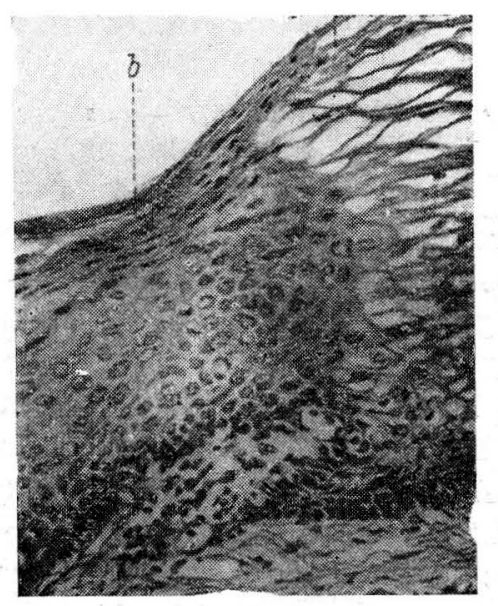

Figura número 1. Rúbrica I

$=$ Con desarrollo de las papilas:

Rúbrica II.-Desarrollo de las papilas hacia la periferia $\ldots \ldots \ldots \ldots \ldots$ II-a 


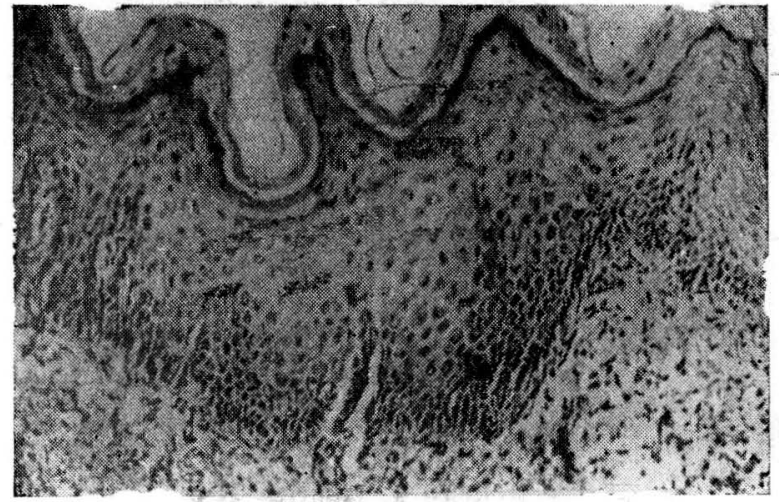

Figura número 2. Rúbrica II-a

Desarrollo de las papilas hacia el corion....................... II-b

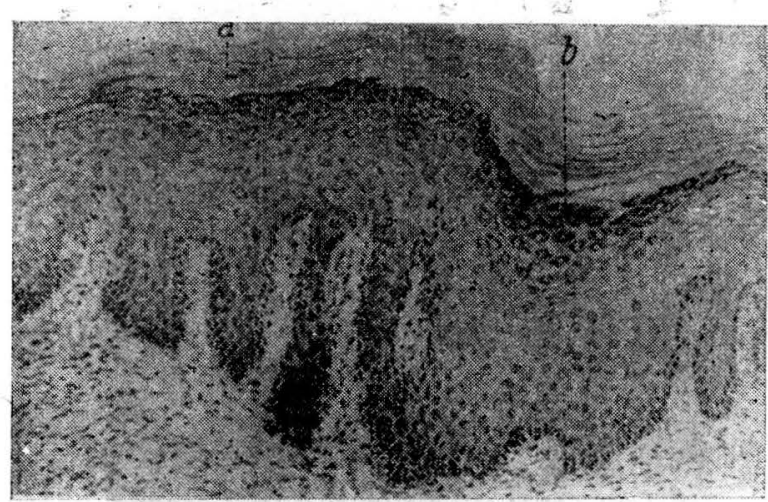

Figura número 3. Rúbrica II-b

Desarrollo de las papilas hacia las glánoulas $\ldots \ldots \ldots \ldots \ldots \ldots \ldots$ II-c 


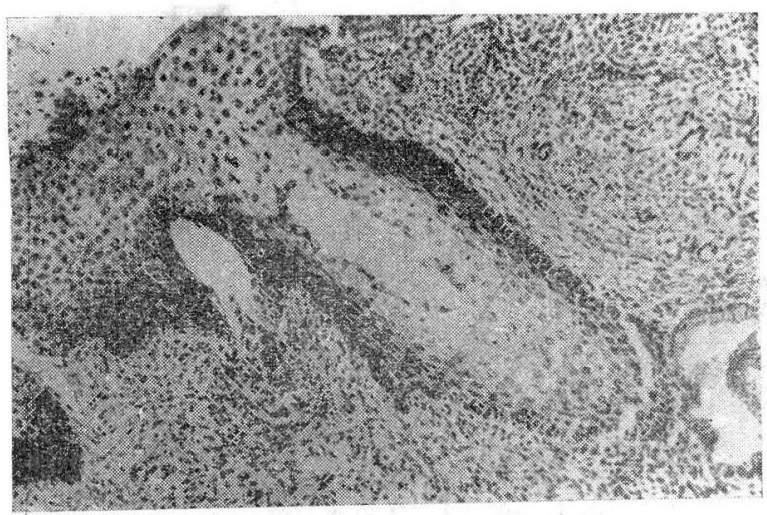

Figura número 4. Rúbrica H-c

Epitelio atípico complejo con tendencia queratinisante.

$=$ En extensión de superficie:

Rúbrica III.-El epitelio de revestimiento está compuesto de capas que pueden ser eliminadas. Ausencia de estratificación del epitelio. Aumento manifiesto del número de núcleos con polimorfismo. Reacción de células peque. ñas en el conjuntivo (Se diferencia principalmente de la Rúbrica 1 por la neoformación celular.

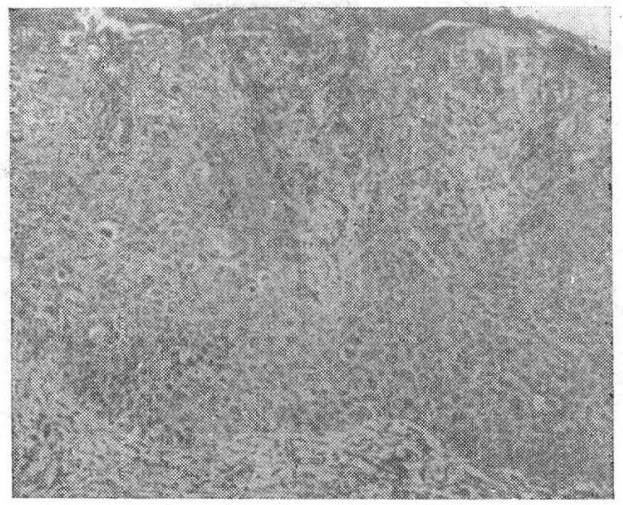

Figura número 5. Rúbrica III

$=$ Con desarrollo de las papilas. 


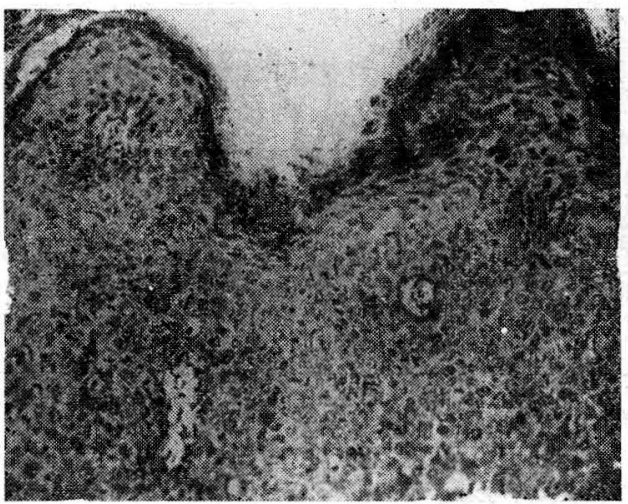

Figura número 6. Rúbrica IV-a

Rúbrica IV.-Desarrollo de las papilas hacia la.periferia........ IV-a Desarrollo de las papilas hacia el corion................. IV-b

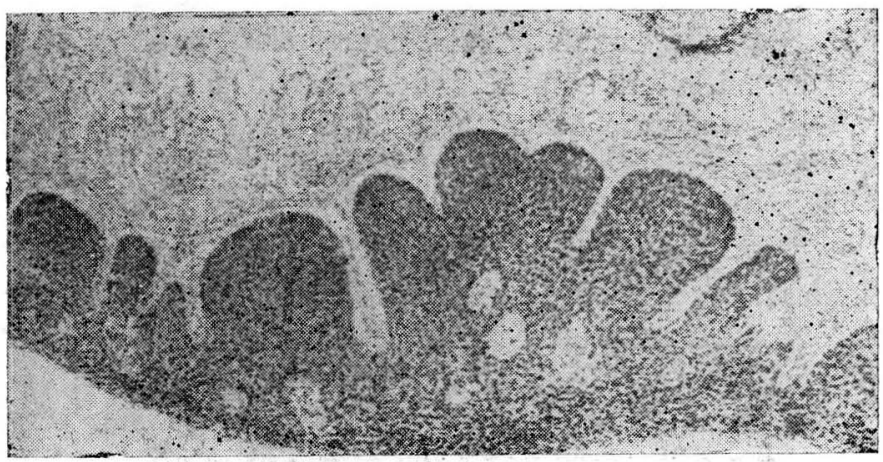

Figura número \%. Rúbrica IV-b

Desarrollo de las papilas hacia las glándulas $\ldots \ldots \ldots \ldots \ldots \ldots$ IV-e 


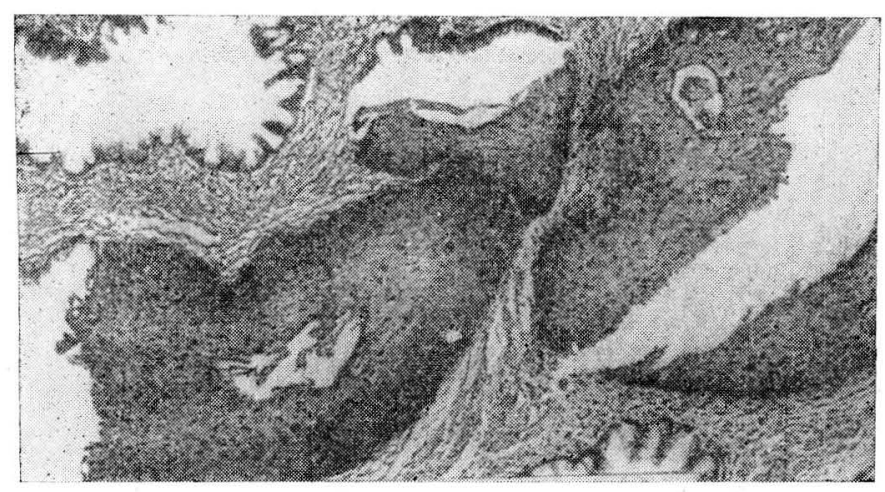

Figura número 8. Rúbrica IV-e

Como lo comentó uno de nosotros en un trabajo publicado hace varios años, las Rúbricas III y IV presentan una estructura histológica característica; se ha perdido por completo la estrati. ficación normal, todo el espesor está formado por células inma. duras con irregularidades celulares y nucleares; se observan abun. dantes mitosis atípicas, es muy marcada la alteración de la rela_ ción núcleo_protoplasmática, y a veces se observa capa córnea, éste es el epitelio exacerbado de los autores alemanes y correspon. de al carcinoma intra_epitelial, carcinoma superficial, carcinoma in situ de los autores americanos y franceses. Actualmente se ha_ lla claramente demostrado que el "epitelio exacerbado" es en rea. lidad un carcinoma de desarrollo superficial incipiente que pre. senta la membrana basal intacta, concepto éste de Roberto Me. yer y que se halla en completo desacuerdo con el postulado de Virchow, quien sostenía que solo podía considerarse como carcino_ ma aquellas alteraciones celulares epidérmicas que hacian su cre. cimiento con invasión hacia el corión, destruyendo la membrana basal.

Comentan los ponentes en seguida de la descripción de los epitelios, el problema de la interpretación exacta del carcinoma intra_epitelial o in_situ, y nos dan el concepto de Ios Laborato_ rios de la Clínica Ginecológica de París, y el de Memoir Marsh; creemos que los conceptos por ellos emitidos son sustancialmente los mismos sostenidos por Roberto Meyer. Sin embargo, juzgamos que las anomalias en el número y en la disposición de los cromo. somas descrita por Moricard y presentada en el Congreso Internacional de Obstetricia y Ginecología de Ginebra deben ser te. nidas en cuenta; ellos se refieren a la gran cantidad die células que 
presentan sus cromosomas en metafase (más del 50\%), con ano. malías en su posición y en su número (más del 10\%), caracterís ticas éstas que no se encuentran en las otras lesiones cervicales, siendo esto efecto y no causa del carcinoma intra_epitelial, como lo señala el autor.

Con gran satisfacción vemos que nuestros colegas de Bogotá, hacen un justo elogio al test de Schiller y a la importancia del estudio del glucógeno en las preparaciones microscópicas, creemos sinceramente que su estudio es un gran paso en el difícil proble. ma de los epitelios atípicos del cervix, y en nusstra cátedra de ginecología no vacilamos en enseñar y preconizar lo expuesto por los ponentes, que es el fiel reflejo del pensamiento europeo. Sin embargo, creemos que debe tenerse siempre presente al hacer un estudio del cervix, lo demostrado por el doctor Hans Limburg, di la Universidad de Hamburgo, de que algunos carcinomas de alta diferenciación celular contienen gran cantidad de glucógeno y son por consiguiente yodo positivos; esto si bien en parte le res. ta un poco de valor al Schiller, en nada perjudica el estudio de las preparaciones microscópicas; ya que el mismo doctor Limburg nos dice que son carcinomas de alta diferenciación celular. Tam. bién ha demostrado este tumor que en algunos casos el lugol altera, en parte, los epitelios y ello, si no lo tenemos en cuenta, pue. de inducirnos a error al leer los cortes histológicos.

En la segunda parte de la ponencia los doctores de Cundina. marca nos presentaron un resumen de 21 casos de carcinoma intraepitelial, y al margen el comentario de 4 casos no incluí. dos por las razones por ellos anotadas.

Por carecer de los informes anatomopatológicos de cada caso, del número de cortes efectuados a cada espécimen, y de sus co_ rrespondientes imágenes que los ponentes por incovenientes se. guramente mayores, no pudieron remitir con el trabajo, nos abs. tenemos a comentar los casos separadamente.

Queremos solo resaltar el hecho, que bien merece tenerse en cuen $\downarrow$ a, de que en uno de ellos la citología fue negativa antes del tratamiento de la lesión, dato éste opuesto a la positividad de los extendidos vaginales del carcinoma intra.epitelial encontrado por Funck Brentano y sus colaboradores, y al hallado por uno de nosotros en el trabajo anteriormente citado.

En la tercera parte se hicieron comentarios y se sacaron con. clusiones relacionadas con el diagnóstico y la conducta que ha de seguirse en el carcinoma intra_epitelial 
Primero los autores se refieren al término que se debe preci. sar para esta entidad, y se muestran con lógica razón partidarios del término carcinoma intra_epitelial, pues juzgan que se debe de preferir el concepto histopatológico. Estamos seguros de que la gran mayoría de los médicos participantes en esta III Con. vención de Obstetricias y Ginecología acogen gustosos el tér mino propuesto por los doctores de Bogotá, y que $\epsilon_{s}$ el mismo adoptado por la Clínica Ginecológica de París que dirige el pro_ fesor Funck Brentano.

En seguida se refirieron los ponentes al arduo problema del diagnóstico del carcinoma intra_epitelial y nos precisaron las cin. co características que ellos juzgaron indispensables para el diagnóstico de esta entidad, desde el punto de vista histológico; noso_ tros una vez más estamos identificados con el pensamiento de los firmantes de la ponencia y comprendemos el que hayan omi. tido la característica de la membrana conjuntiva basal intacta por considerar que su sola enumeración era ya un pleonasmo.

Al margen de su definición y su diagnóstico en cuanto la se. paran de las lesiones displásicas, quenemos hacer un breve co. mentario en lo referente a los factores esenciales para poder con. siderar un carcinoma intra_epitelial como tal, es decir, no inva. sor. Creemos que con el fin de tener unidad de criterio mundial, es necesario el que se fije un número determinado de cortes his. tológicos por milímetros; y cuánto es el mínimo de tejido sano necesario que debe ser inclúdo, y cuántos cortes deben de efec. tuarse en él para considerar que una lesión es in.situ. Estamos intimamente convencidos de que mientras no se logre esta uni. dad de concepto, es imposible el establecer relaciones entre tra bajos sobre el mismo tema y sacar conclusiones sobre el "quid" más importante que es tratamiento, es decir, la conducta a se. guir, ya que todos tendrán como base las apreciaciones subjeti. vas y ello por definición impide la unidad de criterio.

En seguida los autores nos comentan de nuevo el difícil diagnóstico del carcinoma intra_epitelial en el embarazo y la conduc. ta que ha de tomarse en su presencia; nos parece admirable y plausible el pensamiento de los doctores bogotanos, pues solo con precaución y reserva, tanto en el embarazo como en el post_partum podremos estar en posesión de la verdad respecto a su diagnós. tico y como consecuencia de ello, en la ruta certera para la con. ducta terapéutica.

A continuación se preguntan los ponentes si el carcinoma intra_epitelial evoluciona hacia un carcinoma invasor, y sí el in. 
vasor se encuentra siempre precedido de uno intra_epitelial. Creemos, como lo expusimos un poco más atrás, que mientras no se establezca una unidad mundial en la cantidad de tejido adya. cente que debe de incluirse, y el número de cortes que deben de efectuarse por unidad de volumen, no es posible dar una respues_ ta cierta basados en el sumum de la verdad que es la experiencia, pues estaremos juzgando y sacando conclusiones de casos de di. ferentes grados clínicos.

Y lo mismo podemos decir referente a la incidencia, de allí el que nos expliquemos las variaciones de los porcentajes de $1.8 \%$ de Marsh y Hellman, de $1.2 \%$ de Funck Brentano, de $0.57 \%$ de Winship y de $0.25 \%$ de los ponentes.

Finalmente se nos expuso en el excelente trabajo que acaba. mos de escuchar un admirable resumen respecto al tratamien. to a seguir en las diferentes edades y estados de las pacientes. Una vez más estamos identificados con el pensamiento de los po. nentes en lo referente al tratamiento del carcinoma intra_epite. lial en las embarazadas, en las premenopáusicas yen las que no pueden someterse a control, solo discernimos en las pacientes que pueden ser controladas, que en nuestro concepto mientras no se tenga la tan recalcada unidad y veamos la evolución en ellas, de. bemos ser partidarios de la histerectomía con o sin oforectomía, tal como lo preconizan Meigs y Te Linde, citados en la ponencia y como lo rectifica éste último en el American Journal de Obs. tetricia y Ginecología, de octubre de este año.

Queremos antes de terminar felicitar una vez más a los doc_ tores Guillermo López Escobar, Luis A. Urdaneta, Enrique Dar. nalt, Germán Jordán, Hernando Osorio, Hugo Quijano y Jaime Salazar por el admirable trabajo que nos han presentado, y en nombre de todos los convencionistas, y en el nuestro, damos las gracias a los doctores ponentes por las magnificas enseñanzas que $\tan$ generosamente nos ha dado. 